\title{
Motion Planning for Dual-arm Assembly of Ring-shaped Elastic Objects
}

\author{
Ixchel G. Ramirez-Alpizar, Kensuke Harada, and Eiichi Yoshida
}

\begin{abstract}
This paper discusses a motion planning method for assembling a ring-shaped elastic part to a cylindrical part by using a dual-arm manipulator. When assembling a ringshaped elastic part, it is important to keep the amount of elastic deformation as small as possible during the assembly task. To achieve this purpose, we show that it is effective to utilize the Covariant Hamiltonian Optimization and Motion Planing (CHOMP) method. We introduce an energy based objective functional that is obtained by adding a term related to the potential energy of the elastic object. This objective functional yields a trajectory where the deformation of the elastic object is minimized. At the same time, we modified the collision cost function such that the robot can approach to the assembly parts as much as permitted without colliding. To confirm the validity of the proposed planner, simulation results of the PR2 robot handling a ring-shaped elastic object are shown. We show that our modified motion planner is able to find a feasible trajectory keeping the deformation of the elastic object small, and we analyzed the objective functional weights to obtain smaller energy costs.
\end{abstract}

\section{INTRODUCTION}

The robotic manipulation of flexible objects remains a challenging topic, due to the complex dynamics inherent to its physical properties in comparison with rigid objects. In the last decades, the number of industrial robots used at factories has been increasing. However, there are still a number of assembling processes in which most of the work remains done by humans. The reason is that humans possess (or are able to get) the dexterity required to handle objects of several types of materials, shapes, sizes, etc. In this work, we consider the assembly process of a ring-shaped elastic object into a cylinder. This type of assembly is commonly carried out in the manufacturing process of machines in order to seal oil or water pipes. When a human does the assembly process described above, usually he/she will first insert a part of the ring-shaped object at one side of the cylinder, then he/she will pull the object, resulting in the object's deformation, finally he/she will finish inserting the rest of the object by moving his/her hands towards the opposite side (of the starting position) of the cylinder, as shown in Fig. 1. The goal of this work is to develop a planner able to automatically plan the motion for a dual-arm manipulator that assembles an elastic object into a cylinder, under the assumption that the

I. Ramirez-Alpizar and K. Harada are with the Vision and Manipulation Research Group, Intelligent Systems Research Institute, National Institute of Advanced Industrial Science and Technology (AIST), 1-1-1 Umezono, Tsukuba, 305-8568, Japan \{ixchel.ramiresuarupi-saru, kensuke.harada\}aist.go.jp

E. Yoshida is with the AIST-CNRS JRL (Joint Robotics Laboratory), UMI3218/CRT, 1-1-1 Umezono, Tsukuba, 305-8568, Japan e.yoshidalaist.go.jp

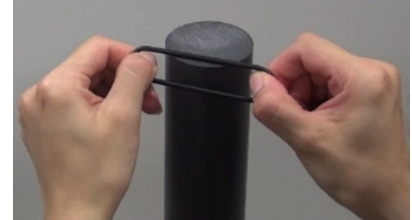

(a) Step 1

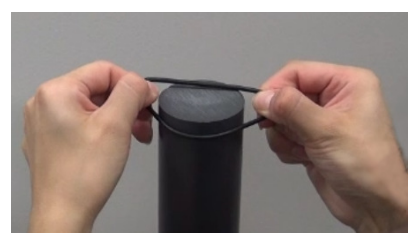

(c) Step 3

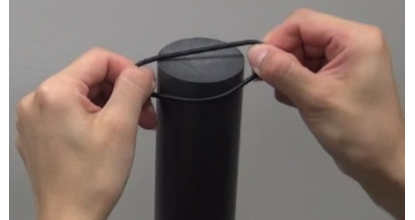

(b) Step 2

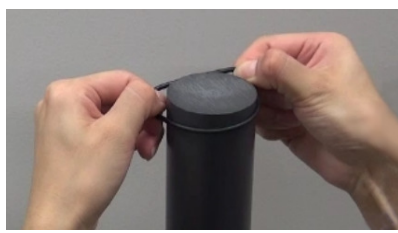

(d) Step 4
Fig. 1. Assembly process of a ring-shaped elastic object performed by a human.

object's material, size and shape are known. To achieve this goal, we have to solve the following two problems: first, to achieve the assembly of a ring-shaped elastic object as shown in Fig. 1, a series of key poses for a robot has to be determined. Second, we have to plan the motion of the robot connecting two key poses. In this paper, we will focus particularly on the second issue.

The objects used at this kind of assembly process, usually have a relatively low elasticity which implies that a large deformation requires a considerable amount of force applied by the manipulator. Also, even if the manipulator could apply large amounts of force, this is not desired as it could lead to the fracture of the manipulated object. Consequently, it is desirable to keep the object's deformation as small as possible. For this problem, this paper proposes a method that can easily and effectively reduce the amount of elastic deformation during the assembly process.

Previous work on manipulation of deformable objects has developed motion planners considering the object's deformation [9], [10]. Lamiraux et al. proposed a motion planner [9] based on a Probabilistic Roadmap (PRM) [11]. However, the assembly of elastic parts has not been considered. Moreover, as PRM planners sample the configuration space of the robot and are typically composed of two phases (finding a feasible trajectory and then optimization so as to remove unnecessary motions), their computational cost is high. On the other hand, motion planners based on optimization techniques have been proposed in recent years. Ratliff et al. proposed an algorithm, which they call Covariant Hamiltonian Optimization and Motion Planing (CHOMP). This method searches a smooth 
and collision-free path given two end-points. It will start with the simplest path (e.g. a straight line), and even if this initial path is not collision free, the CHOMP algorithm has prove to find collision-free trajectories [17]. Since CHOMP is an optimization based method, we are free to choose the objective function and the terminal condition of the optimization. Hence, it can be expected that the motion planning for manipulating elastic objects with complex deformations could be easily achieved by adding an elastic energy related term to the objective function used in CHOMP. We can choose a simple objective function so that the computational cost is low.

In this paper, we consider proposing an object's elastic energy related term which will be included into the optimization problem used in the CHOMP method for planning the assembly motion of an elastic ring-shaped object by using a two-arm robot. We also introduce a less strict collision cost function than the originally proposed in [17], which is necessary to allow the manipulator to be as near as possible to the cylinder without colliding with it. Through simulation analysis, we show that the assembly of the ring-shaped object with a cylinder was achieved. Then, we verify the validity of the collision cost function proposed, and confirm that the planner is able to find a collision-free trajectory. Finally, we show that the addition of the energy related term to the original CHOMP planner, indeed minimizes the deformation of the ring-shaped object and analyzed the influence of the optimization weights in the final trajectory.

This paper is organized as follows: in section II, we briefly review related work. In section III, we give a brief introduction to the CHOMP method and we introduce an energy objective functional to minimize the deformation of the object. In section IV, we show the simulation results of the developed planner using the PR2 robot. In section V, we give the conclusion of this work.

\section{RELATED WORK}

Until now, there have been a number of works on manipulating flexible linear objects, such as ropes, cables, etc. Yamakawa et al. have discussed the motion planning for knotting linear flexible objects, where a model for the linear object is derived and used for the motion planning of the robot to dynamically knot the object [1], and for the deformation control of a flexible rope [2]. Vinh et al. have shown a strategy for knotting a deformable rope using a Wii controller as teaching pennant [3]. Wakamatsu et al. have discussed manipulation plans for knotting and unravelling linear objects by representing the object as a series of crossing states, and based on these states the manipulation plan is determined [4], [5]. Saha and Isto have discussed the motion planning for knotting linear deformable objects around static objects using what they called "topologically biased" probabilistic roadmap in the configuration space of the linear deformable object [6]. Matthews and Bretl have discussed the quasi-static manipulation of planar elastic rods using a sampling-based planning algorithm where the planar elastic rod has a fixed end and the other is held by a robotic gripper [7]. McCarthy and Bretl have discussed the manipulation of planar elastic kinematic chains that have a fixed base and linearly-elastic torsional springs at each joint [8].

Regarding assembly tasks of flexible parts, previous work has mainly discussed the insertion of a flexible beam [12] and a flexible wire [13]. The insertion of a vibrating linear deformable object into a hole by using a force/torque sensor mounted on the robot's wrist has been studied in [16]. The assembly of a rubber belt and fixed pulleys, where a rubber belt is inserted into a small pulley, and then the belt is stretch so as to be inserted into a bigger pulley, have been discussed in [14], [15].

As far as we know, there has been no work on the assembly motion planning of a ring-shaped elastic object. Also, there has been no work on manipulation planning where the CHOMP algorithm was used and the elasticity of the manipulated object was considered.

\section{MOTION Planning}

In this section we explain the motion planning method for the assembly process of elastic objects. First, we made some assumptions about the assembly process. Next, we give a brief introduction to the CHOMP algorithm used for motion planning. Then, we introduce the energy objective functional added to CHOMP so as to minimize the deformation of the ring-shaped object. Finally, we introduce a less strict collision cost function that allows the robot to manipulate the ring-shaped object near the cylinder.

\section{A. Assumptions}

Let us consider the motion planning of the assembly of a ring-shaped object into a cylinder, as shown in Fig. 1. We assume that the diameter of the ring-shaped object without deformation is smaller than that of the cylinder, this implies that the object needs to be stretched in order to be inserted into the cylinder. Furthermore, we make the following assumptions:

1: The manipulator grasps firmly the elastic object.

2: The grasping point of the elastic object with respect to the manipulator's wrist coordinate system is known and it is constant.

3: The size, shape and Young's Modulus of the object are known.

4: The cylinder use for the assembly task is static, rigid and its position and dimensions are known.

We use a dual-arm manipulator to achieve the assembly. Similar to the assembly motion done by a human shown in Fig. 1, the robot first inserts a part of the ring-shaped object at one side of the cylinder, then the robot pulls the object, resulting in the object's deformation, finally the robot finishes inserting the rest of the object by moving the hands towards the opposite side of the starting position. We divide the assembly process in several steps where only one of the arms moves at each step. We generate a set of key poses of a robot by dividing the assembly process into several steps. While a set of key poses are generated automatically, the 


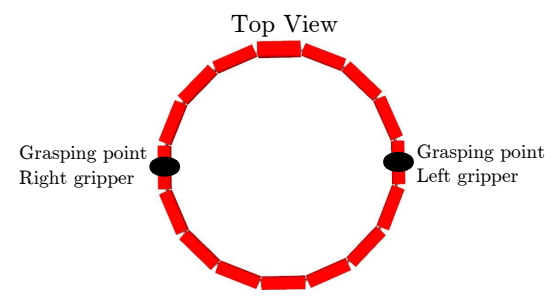

Fig. 2. Grasping points of the ring.

method for generating the key poses is out of the scope of this paper and will be discussed in detail in another paper. Rather, we focus on the motion planning connecting two key poses by using CHOMP, as detailed in the following subsections. For now, we would like to point out that the key poses are generated based on the position of both grippers at each assembly step, which gives robustness to our planner in the sense that even if the gripper does not arrive to the exact desired position, the positioning error will not accumulate. However, it may need to make some extra steps to finish the assembly process correctly.

\section{B. CHOMP Algorithm}

In this work we proposed a motion planning algorithm for manipulating elastic objects, based on the trajectory optimization algorithm developed by Ratliff et al. which is called Covariant Hamiltonian and Motion Planning (CHOMP) [17]. This algorithm is capable of finding a smooth and collisionfree trajectory $\xi$ between two specific configurations $q_{0}$ and $q_{\text {goal }}$ of the configuration space $\mathbb{R}^{m}$. The trajectory $\xi$ is expressed as a function mapping time to robot configurations $q \in \mathbb{R}^{m}$. Using a uniform discretization of $n$ time steps of length $\Delta t$, the trajectory is represented as $\xi \approx\left(q_{1}^{T}, q_{2}^{T}, \ldots, q_{n}^{T}\right)^{T} \in \mathbb{R}^{n \times m}$. The CHOMP algorithm typically starts with a simple straight line between the given configurations (even if it is not collision-free), as the initial trajectory $\xi_{0}$. Then, it optimizes the initial trajectory through an iterative update rule [18] given by:

$$
\xi_{i+1}=\xi_{i}-\frac{1}{\eta}\left(K^{T} K\right)^{-1} \bar{\nabla} \mathcal{U}\left(\xi_{i}\right)
$$

where $\xi_{i}$ is the refined trajectory at iteration $i, K$ is a finite differencing matrix, $\eta$ is the regularization coefficient that determines the trade-off between minimizing the objective functional $\mathcal{U}$ and the step size, and $\bar{\nabla}$ is the functional gradient operator. The objective functional $\mathcal{U}\left(\xi_{i}\right)$ is given by:

$$
\mathcal{U}(\xi)=\mathcal{F}_{\text {obs }}(\xi)+\lambda \mathcal{F}_{\text {smooth }}(\xi)
$$

where $\mathcal{F}_{\text {smooth }}$ is the smoothness objective that penalizes the trajectory $\xi_{i}$ based on dynamical parameters such as the squared velocity norms over the trajectory, and $\mathcal{F}_{\text {obs }}$ is the obstacle objective which penalizes the robot for being near and/or in contact with the environment.

\section{Energy Objective Functional}

In this work we employ CHOMP for the assembly manipulation of a ring-shaped elastic object. We suppose an assembly task as shown in Fig. 1. The objects used at this kind of assembly process, usually have a relatively low elasticity which implies that a large deformation requires a considerable amount of force applied by the manipulator. Consequently, it is desirable to keep the object's deformation as small as possible. In order to minimize the object's deformation, we introduce an energy objective functional that penalizes the trajectory $\xi_{i}$ for deforming the object. Therefore, the objective functional in equation (2) is rewritten as

$$
\mathcal{U}(\xi)=w_{c} \mathcal{F}_{\text {obs }}(\xi)+w_{s} \mathcal{F}_{\text {smooth }}(\xi)+w_{e} \mathcal{F}_{\text {energy }}(\xi)
$$

where $w_{c}, w_{s}, w_{e}$, are the weights of the obstacle objective, the smoothness objective and the energy objective, respectively. We employ the smoothness objective as define in [18], and the obstacle objective as described in section III-D. The energy objective functional $\mathcal{F}_{\text {energy }}$ is given by

$$
\mathcal{F}_{\text {energy }}(\xi)=\int_{0}^{1} U(\xi(t))\left\|\frac{d}{d t} \xi(t)\right\| d t
$$

where $U(\xi)$ is the energy cost function in the configuration space.

The functional gradient [19] of the energy objective is obtained as

$$
\bar{\nabla} \mathcal{F}_{\text {energy }}(\xi)=\left\|\xi^{\prime}\right\|\left(\left(I-\hat{\xi}^{\prime} \hat{\xi}^{\prime T}\right) \nabla U-U \kappa\right)
$$

where $\kappa$ is the curvature vector [19] given by

$$
\kappa=\frac{\xi^{\prime \prime}}{\left\|\xi^{\prime}\right\|^{2}}\left(I-\hat{\xi}^{\prime} \hat{\xi}^{\prime T}\right)
$$

$\nabla U$ is the gradient of $U$, and $\xi^{\prime}$ and $\hat{\xi}$ denote the time derivative and the normalized vector of $\xi$, respectively.

In this research, we consider modeling the elastic ring by using a set of rigid bodies connected through springs. For the energy cost function $U(\xi)$, we use the potential energy of a spring,

$$
U(\xi)=\frac{1}{2} k x_{d}(\xi)^{2}
$$

where $k$ is the ideal stiffness of the spring, and $x_{d}(\xi)$ is the deformation of the spring. In this case, we approximate the stiffness of the ring-shaped object through its Young's modulus (tensile stress by tensile strain) $E$ as

$$
k=\frac{E A_{o}}{L_{o}}
$$

where $A_{o}$ is the original cross-sectional area of the object, and $L_{o}$ is the original length of the object. The deformation of the ring-shaped object is calculated approximatly through the position of the robot arms' wrists, under the assumption that the robot grasp the ring firmly.

\section{Collision Cost Function}

Next, we consider modifying the collision cost function such that the CHOMP algorithm can be used for the assembly motion planning of elastic parts. The obstacle objective in equation (3) is given by [17]

$$
\left.\mathcal{F}_{\text {obs }}(\xi)=\int_{0}^{1} \int_{\mathcal{B}} c(x(\xi(t), u)) \| \frac{d}{d t} x(\xi(t), u)\right) \| d u d t
$$




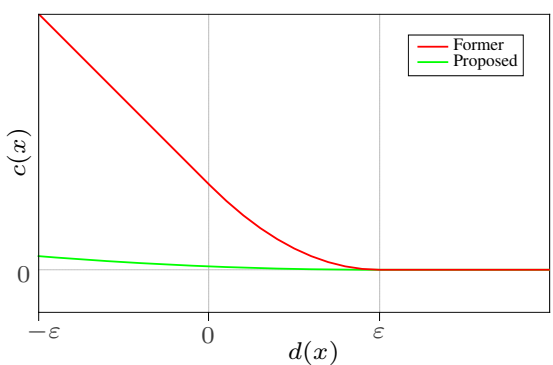

Fig. 3. Comparison between collision cost functions $c(x)$, when $d_{\text {clear }}=$ $\varepsilon$.

where $\mathcal{B} \subset \mathbb{R}^{3}$ is the set of points on the exterior body of the robot and $x$ denotes the forward kinematics mapping a robot configuration $q$ and a particular body point $u$ to a point $x(q, u)$ in the workspace, and $c$ is a workspace collision cost function that penalizes the robot for being inside or near the environment, and given as

$$
c(x)=\left\{\begin{aligned}
-d(x)+\frac{1}{2} \varepsilon, & \text { if } d(x)<0 \\
\frac{1}{2 \varepsilon}(d(x)-\varepsilon)^{2}, & \text { if } 0 \leq d(x) \leq \varepsilon \\
0, & \text { otherwise }
\end{aligned}\right.
$$

where $d(x)$ is the distance from a point $x$ to the boundary of the nearest obstacle and $\varepsilon$ is the collision threshold. Here, we would like to emphasize that the collision cost function is given in the workspace, since it evaluates the distance between the robot body and the environment. However, in the case of the energy cost in equation (6), as we seek to minimize the object's deformation, we use the configuration space to directly obtain the position of both grasping points and evaluate the object's deformation.

In section IV we show that using the collision cost function of equation (9) yields indeed a collision free trajectory. However this collision cost pushes away the trajectory needlessly far from a possible collision with the environment (cylinder), thus stretching the ring-shaped object more than two times its original size which may result in the undesired realease of the ring-shaped object and failing the assembly task.

In our case, as we want to avoid collisions with the environment and at the same time minimize the deformation of the elastic object, we need to have a balance between the collision cost function and the energy cost function discussed in section III-C. For this reason, we define the collision cost function as follows,

$$
c(x)=\left\{\begin{aligned}
\frac{1}{2} \varepsilon(d(x)-\varepsilon)^{2}, & \text { if } d(x)<d_{\text {clear }} \\
0, & \text { otherwise }
\end{aligned}\right.
$$

where we define $d_{\text {clear }}$ as the minimum distance between the boundary of a body point and the boundary of the nearest obstacle, before being in collision. With this collision cost function, the robot is able to move as near as allowed by $d_{\text {clear }}$ to the environment.

Fig. 3 shows the plot of the original collision cost function given by equation (9) and the plot of the proposed collision cost function given by equation (10), for a fair comparison we let $d_{\text {clear }}=\varepsilon$. Note that if $d_{\text {clear }}<\varepsilon, c(x)$ will become zero faster, but the rest of the function is the same as the

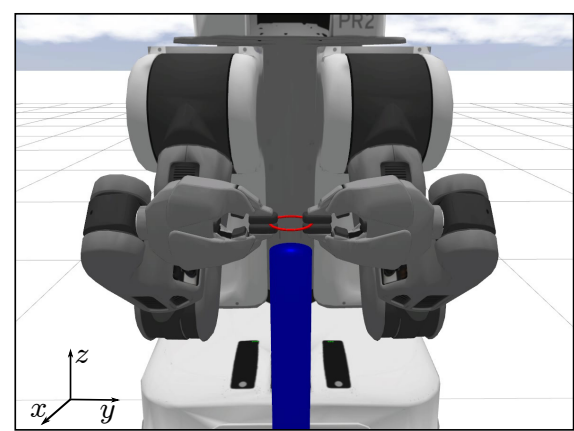

Fig. 4. Initial state of the PR2 robot holding the ring-shaped object.

one depicted in Fig. 3. It can be seen that the original cost function rapidly increases as the distance to the obstacle gets smaller, while the proposed cost function increases with a difference of $1 / \varepsilon^{2}$ times the original one (when $d(x)<0$ and $\varepsilon<1$ ). Therefore, even for very small values of $\varepsilon$ (e.g. $1 \times 10^{-6}$ ) the value of $c(x)$ when $d(x)>0$, will no change significantly. Only when the robot is not in collision $(d(x)>$ 0 ) we could get similar values of $c(x)$ for the original cost function using small values of $\varepsilon$ and the proposed one.

\section{Simulation Results}

\section{A. Simulation setting}

The Robot Operating System (ROS) platform and the Gazebo simulator are used to carry out the simulation of the PR2 robot handling an elastic object. The ring-shaped elastic object is approximated by rigid cylindrical links connected by joints that have spring-damper system properties, as shown in Fig. 2. This approximated model is merely for visual purposes, as we are not using any kind of visual nor sensor feedback. However, it should be pointed out that the approximation of deformable objects by rigid links connected by joints with spring-damper properties, has demonstrated to give a fair approximation of the behavior of the real object [20]. We consider the assembly of a ring-shaped object into a cylinder located at the center between the arms of the robot. Fig. 4 shows the initial state from which the assembly process starts. The ring-shaped elastic object has an inner radius of $49.9 \mathrm{~mm}$, a thickness of $3 \mathrm{~mm}$ and a Young's modulus of $E=4.125 \mathrm{MPa}$. The cylinder has a radius of $50 \mathrm{~mm}^{1}$

Fig. 5 shows the simulation results of the assembly process carried out using the proposed motion planner, where each snapshot shows the achieved position at each assembly step by the PR2 robot. As mentioned in section III-A, the assembly process is accomplished in 6 steps, and it begins by moving the left arm at step 1 (Fig. 5(a)), and finishing with the right arm at step 6 (Fig. 5(f)). It can be seen that the robot's arms move about one third of the cylinder's diameter in the $x$ direction at each step.

\footnotetext{
${ }^{1}$ In this particular case the difference between the cylinder's diameter and the ring's one is $0.1 \mathrm{~mm}$. Nevertheless, for larger differences it can be expected that the assembly process would need more steps to be accomplished, as the number of steps depends on the diameters of both the object and the cylinder.
} 
(a) Step 1

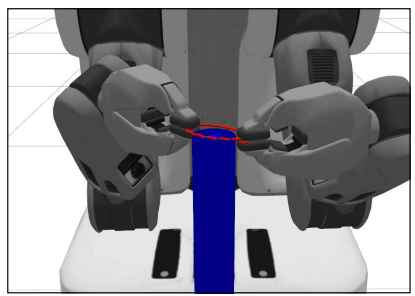

(c) Step 3

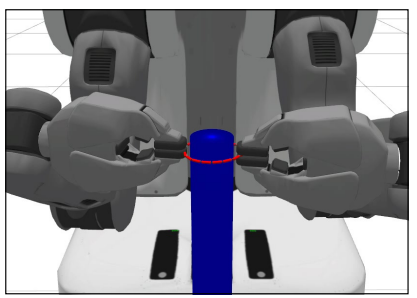

(e) Step 5

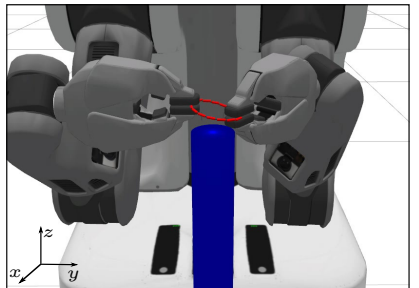

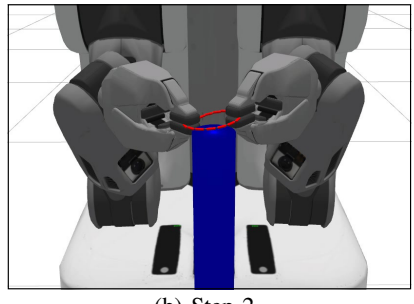

(b) Step 2

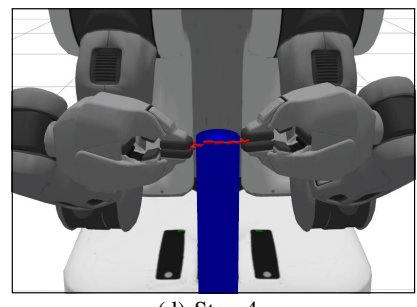

(d) Step 4

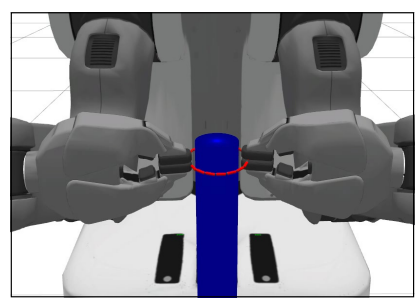

(f) Step 6
Fig. 5. Assembly process.

Let us discuss the importance of the collision cost function, for this purpose we set $w_{e}=0$ in equation (3) (no consideration of the object's deformation). Fig. 6 shows the top view (X-Y plane) in (a) and the front view (Y-Z plane) in (b), of the trajectory described by the grasping position of the left gripper. Here, we compare the trajectory obtained using the original CHOMP's collision cost in equation (9) which we call "former", and the trajectory obtained using equation (10) which we call "proposed". The continuous line in Fig. 6 represents the perimeter of the cylinder. As a first attempt we choose a collision weight of $w_{c}=10.0$ which yields a collision cost smaller than the original one, as discussed at the end of section III-D. It can be seen that the grasping position trajectory obtained from the original CHOMP makes a big turn around the cylinder, while the trajectory obtained through the proposed collision cost function considerably shortens the turn around the cylinder. We must point out that for some of the assembly steps, when the given gripper position is very close to the cylinder, the original CHOMP was not able to yield a collision-free trajectory (using the same collision threshold $\varepsilon$ ), since pulling the trajectory far from the object implies a considerable increase in the length of the trajectory therefore increasing its cost. In contrast, with the proposed collision cost we were able to get a collision-free trajectory, as the proposed cost function pulls the trajectory by a smaller amount at each iteration, keeping the length of the trajectory smaller. Despite this, the average number of iterations needed was the same or less than with the original CHOMP.

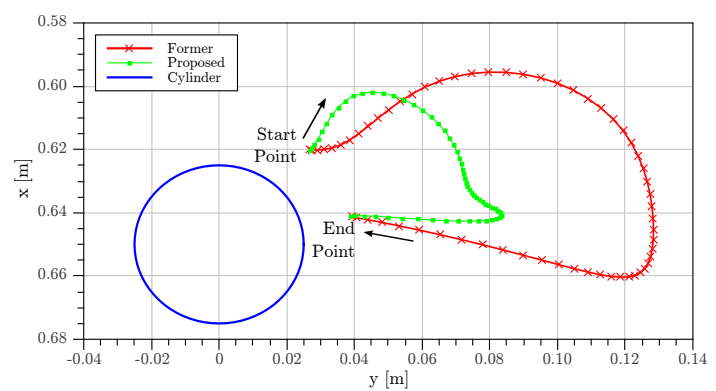

(a)

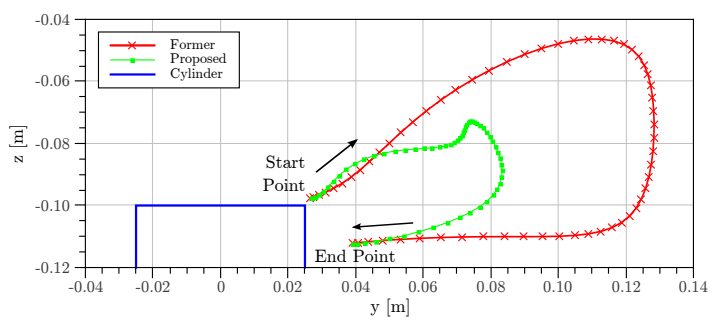

(b)

Fig. 6. Trajectory of the grasping point of the left arm's gripper obtained with the original CHOMP's collision cost function given by equation (9) and the proposed collision cost given by equation (10). In (a) the X-Y plane and in (b) the Y-Z plane.

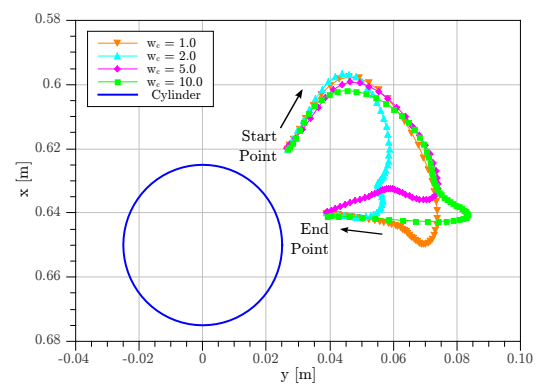

(a)

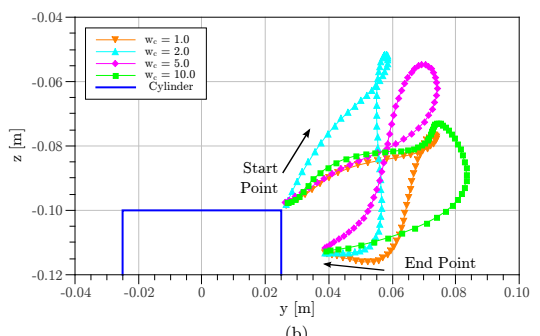

Fig. 7. Trajectory of the grasping point of the left arm's gripper using the proposed collision cost function for 4 different collision weights. In (a) the $\mathrm{X}-\mathrm{Y}$ plane and in (b) the Y-Z plane, for $w_{e}=0$.

\section{B. Analysis of Objective Functionals' Weights}

Next, we analyzed the influence of the collision weight cost $w_{c}$ in the resulting trajectory $\left(w_{e}=0\right)$. Fig. 7 shows the top view (X-Y plane) in (a) and the front view (Y-Z plane) in (b) of the trajectories described by the grasping position of the left gripper, obtained with 4 different collision weights $w_{c}$. It can be seen that the trajectories are similar for all the values of $w_{c}$. Their collision costs, given by equation (8), are also similar (within 0.0001).

Fig. 8 shows the top view (X-Y plane) in (a) and the 

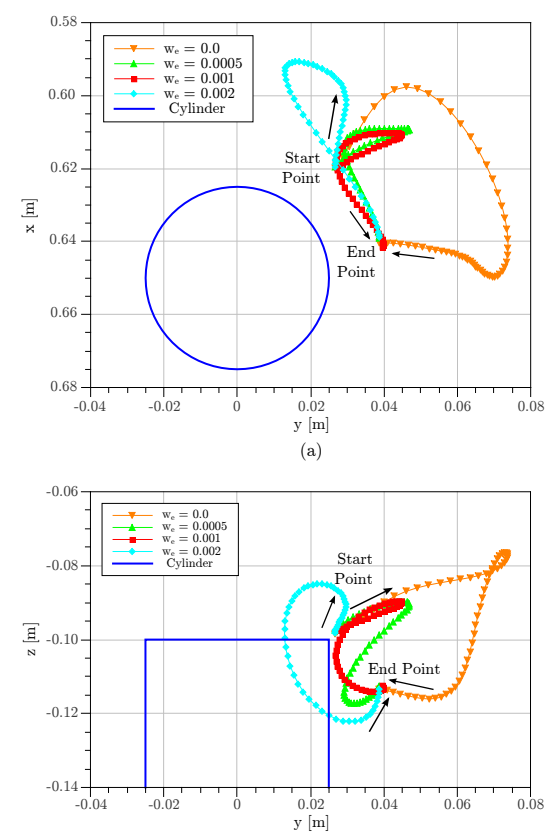

(b)

Fig. 8. Trajectory of the grasping point of the left arm's gripper using the proposed motion planner with and without the energy objective functional. In (a) the X-Y plane and in (b) the Y-Z plane, with $w_{s}=0.00001$ and $w_{c}=1.0$.

front view (Y-Z plane) in (b), of the trajectories described by the grasping position of the left gripper, obtained with the proposed collision cost function $\left(w_{e}=0\right)$, and obtained when adding the energy objective functional to the motion planner $\left(w_{e} \neq 0\right)$. As it can be seen, the trajectories obtained when $w_{e} \neq 0$ are considerably shorter and closer to the cylindrical object than the one with $w_{e}=0$. It can also be observed that for all the values of $w_{e}$ the trajectories are similar. At first sight, it may look like the elastic object is being stretched very much. However, the fact that its moving towards the robot $(x \rightarrow 0)$ means its getting closer to the other gripper, therefore the elastic object is being stretched less than when moving in the opposite direction.

Similarly, Fig. 9 shows the top view (X-Y plane) in (a) and the front view (Y-Z plane) in (b), of the trajectories described by the grasping position of the right gripper, obtained with the proposed collision cost function $\left(w_{e}=0\right)$, and obtained when adding the energy objective functional to the motion planner $\left(w_{e} \neq 0\right)$. At this assembling step, it can be seen that the trajectory obtained with $w_{e}=0.0005$ describes a semicircle just as if it was passing along the cylinder's edge. Consequently minimizing the object's deformation. In contrast, the trajectory obtained with $w_{e}=0$ makes a turn around the cylinder in a similar way to the trajectory at step 3. Similar to the case shown in Fig. 8, for $w_{e}=0.001$ and 0.002 the gripper moves towards the robot, avoiding the cylinder and getting closer to the opposite gripper, reducing the deformation of the elastic object.

For analyzing the influence of the objective functionals weights, several simulations were carried out. The collision cost and energy cost given by equations (8) and (4), respec-

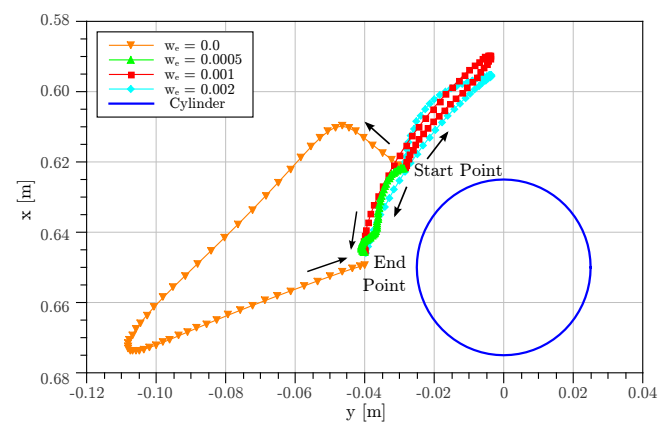

(a)

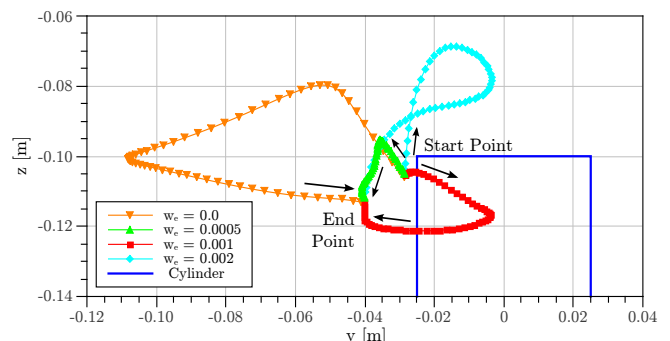

Fig. 9. Trajectory of the grasping point of the right arm's gripper using the proposed motion planner with and without the energy objective functional. In (a) the X-Y plane and in (b) the Y-Z plane, with $w_{s}=0.00001$ and $w_{c}=1.0$.

TABLE I

COLLISION COST AND ENERGY COST FOR $w_{e}=0.0005$

\begin{tabular}{|c|c|c|c|}
\hline$w_{s}$ & $w_{c}$ & $\begin{array}{c}\text { Collision } \\
\text { cost }\end{array}$ & $\begin{array}{c}\text { Energy } \\
\text { cost }\end{array}$ \\
\hline \multirow{3}{*}{0.000005} & 0.5 & 0.0162 & 17.14 \\
& 1.0 & 0.0133 & 25.92 \\
& 2.0 & 0.0135 & 22.91 \\
\hline \multirow{3}{*}{0.00001} & 0.5 & 0.0165 & 21.40 \\
& 1.0 & 0.0136 & 20.92 \\
& 2.0 & 0.0140 & 20.04 \\
\hline \multirow{3}{*}{0.00002} & 0.5 & 0.0155 & 18.34 \\
& 1.0 & 0.0141 & 22.51 \\
& 2.0 & 0.0149 & 25.06 \\
\hline \hline \multicolumn{2}{|c|}{ Average } & 0.0146 & 21.58 \\
\hline
\end{tabular}

tively, for all the assembly process are summarized in Table I for $w_{e}=0.0005$, Table II for $w_{e}=0.001$ and Table III for $w_{e}=0.002$. The smoothness costs are not included as they are in the order of hundreds of thousands. Furthermore the largest difference among them is $0.25 \%$ of the overall average cost, which means there is no significant difference among them. It can be seen, that overall the smallest energy costs were obtained with $w_{e}=0.002$, while the collision costs does not change significantly. The smallest energy cost was obtained for the combination of $w_{e}=0.002$ with $w_{c}=2.0$ and $w_{s}=0.00001$. If we group the resutls by $w_{s}$ instead of $w_{e}$ and calculate the average energy cost, it is found that the smallest energy costs were obtained with $w_{s}=0.00001$. Similarly, if we group by $w_{c}$, it is found that the smallest energy costs were obtained with $w_{c}=2.0$. These results coincide with the smallest energy cost obtained. 
TABLE II

COLLISION COST AND ENERGY COST FOR $w_{e}=0.001$.

\begin{tabular}{|c|c|c|c|}
\hline$w_{s}$ & $w_{c}$ & $\begin{array}{c}\text { Collision } \\
\text { cost }\end{array}$ & $\begin{array}{c}\text { Energy } \\
\text { cost }\end{array}$ \\
\hline \multirow{3}{*}{0.000005} & 0.5 & 0.0153 & 19.42 \\
& 1.0 & 0.0180 & 15.94 \\
& 2.0 & 0.0142 & 17.90 \\
\hline \multirow{3}{*}{0.00001} & 0.5 & 0.0141 & 20.21 \\
& 1.0 & 0.0153 & 16.20 \\
& 2.0 & 0.0144 & 15.67 \\
\hline \multirow{3}{*}{0.00002} & 0.5 & 0.0150 & 20.99 \\
& 1.0 & 0.0155 & 19.73 \\
& 2.0 & 0.0136 & 26.60 \\
\hline \hline \multicolumn{2}{|c|}{ Average } & 0.0150 & 19.18 \\
\hline
\end{tabular}

TABLE III

COLLISION COST AND ENERGY COST FOR $w_{e}=0.002$.

\begin{tabular}{|c|c|c|c|}
\hline$w_{s}$ & $w_{c}$ & $\begin{array}{c}\text { Collision } \\
\text { cost }\end{array}$ & $\begin{array}{c}\text { Energy } \\
\text { cost }\end{array}$ \\
\hline \multirow{3}{*}{0.000005} & 0.5 & 0.0154 & 18.75 \\
& 1.0 & 0.0142 & 17.74 \\
& 2.0 & 0.0167 & 15.33 \\
\hline \multirow{3}{*}{0.00001} & 0.5 & 0.0155 & 17.83 \\
& 1.0 & 0.0155 & 17.16 \\
& 2.0 & 0.0170 & 15.22 \\
\hline \multirow{3}{*}{0.00002} & 0.5 & 0.0163 & 16.88 \\
& 1.0 & 0.0161 & 21.25 \\
& 2.0 & 0.0158 & 16.47 \\
\hline \hline \multicolumn{2}{|c|}{ Average } & 0.0158 & 17.40 \\
\hline
\end{tabular}

\section{Conclusion}

This paper discussed the motion planning of a dual-arm manipulator handling a ring-shaped elastic object towards an assembly task. The main results in this paper are summarized as follows:

1. We introduced an energy objective functional that minimized the deformation of the elastic object when manipulated by a dual-arm robot.

2. We introduced a less strict collision cost function that allows the robot to be near to its environment without colliding. This function proved that it can yield collision-free trajectories.

3. We showed the simulation results to confirm the validity of the proposed motion planner. The addition of the energy functional to the original CHOMP method proved to be effective for minimizing the elastic energy of the object.

4. We analyzed the influence of the objective functionals weights, and found that for the smoothness and collision costs there is no significant difference, while for the energy cost it was found a combination of weights that yields the smallest energy cost.

In the future we would like to discuss in detailed the assembly sequence of elastic ring-shaped objects. Through this research, we can recognize the potential of CHOMP as a manipulation planner for elastic objects where the complex manipulation of this kind of objects can be executed by modifying the original CHOMP method. Application of CHOMP for another style of manipulation of elastic objects is considered to be our future research topic.

\section{REFERENCES}

[1] Y. Yamakawa, A. Namiki, and M. Ishikawa, "Motion planning for dynamic knotting of a flexible rope with a high-speed robot arm," in Proc. of IEEE/RSJ Int. Conf. on Intelligent Robots and Systems, Taipei, Taiwan, 2010, pp. 49-54.

[2] Y. Yamakawa, A. Namiki, and M. Ishikawa, "Simple model and deformation control of a flexible rope using constant, high-speed motion of a robot arm," in Proc. of IEEE Int. Conf. on Robotics Automation, Saint Paul, MN, 2012, pp. 2249-2254.

[3] T. V. Vinh, T. Tomizawa, S. Kudoh, and T. Suehiro, "A new strategy for making a knot with a general-purpose arm," in Proc. of IEEE Int. Conf. on Robotics Automation, Saint Paul, MN, 2012, pp. 2217-2222.

[4] H. Wakamatsu, A. Tsumaya, E. Arai, and S. Hirai, "Planning of onehanded knotting/raveling manipulation of linear objects," in Proc. of IEEE Int. Conf. on Robotics Automation, New Orleans, LA, 2004, vol. 2, pp. 1719-1725.

[5] H. Wakamatsu, A. Tsumaya, E. Arai, and S. Hirai, "Manipulation planning for unraveling linear objects," in Proc. of IEEE Int. Conf. on Robotics Automation, Orlando, Florida, 2006, pp. 2485-2490.

[6] M. Saha and P. Isto, "Manipulation planning for deformable linear objects," IEEE Trans. on Robotics, vol. 23, no. 6, pp. 1141-1150, Dec. 2007.

[7] D. Matthews and T. Bretl, "Experiments in quasi-static manipulation of a planar elastic rod," in Proc. of IEEE/RSJ Int. Conf. on Intelligent Robots and Systems, Vilamoura, Portugal, 2012, pp. 5420-5427.

[8] Z. McCarthy and T. Bretl, "Mechanics and manipulation of planar elastic kinematic chains," in Proc. of IEEE Int. Conf. on Robotics Automation, Saint Paul, MN, 2012, pp. 2798-2805.

[9] F. Lamiraux and L. E. Kavraki, "Planning paths for elastic objects under manipulation constraints," The Int. J. of Robotics Research, vol. 20, no. 3, pp. 188-208, Mar. 2001.

[10] M. Moll and L. E. Kavraki, "Path planning for deformable linear objects," IEEE Trans. on Robotics, vol. 22, no. 4, pp. 625-636, Aug. 2006.

[11] L. E. Kavraki, P. Švestka, J.-C. Latombe, and M. H. Overmars, "Probabilistic roadmaps for path planning in high-dimensional configuration spaces," IEEE Trans. on Robotics and Automation, vol. 12, no. 4, pp. 566-580, Aug. 1996.

[12] Y. F. Zheng, R. Pei, and C. Chen, "Strategies for automatic assembly of deformable objects," in Proc. of IEEE Int. Conf. on Robotics Automation, Sacramento, CA, 1991, vol. 3, pp. 2598-2603.

[13] H. Nakagaki, K. Kitagaki, T. Ogasawara, and H. Tsukune, "Study of deformation and insertion tasks of a flexible wire," in Proc. of IEEE Int. Conf. on Robotics Automation, Albuquerque, NM, 1997, vol. 3, pp. 2397-2402.

[14] J. Miura and K. Ikeuchi, "Assembly of flexible objects without analytical models," in Proc. of IEEE/RSJ Int. Conf. on Intelligent Robots and Systems, Pittsburgh, PA, 1995, vol. 2, pp. 77-83.

[15] J. Miura and K. Ikeuchi, "Task planning of assembly of flexible objects and vision-based verification," Robotica, vol. 16, no. 3, pp. 297-307, May 1998.

[16] S. Yue and D. Henrich, "Manipulating deformable linear objects: sensor-based fast manipulation during vibration," in Proc. of IEEE Int. Conf. on Robotics Automation, Washington, DC, 2002, pp. $2467-$ 2472.

[17] N. Ratliff, M. Zucker, J. A. Bagnell, and S. Srinivasa, "CHOMP: Gradient optimization techniques for efficient motion planning”, Proc. of IEEE Int. Conf. on Robotics and Automation, pp. 489-494, 2009.

[18] M. Zucker, N. Ratliff, A. D. Dragan, M. Pivtoraiko, M. Klingensmith, C. M. Dellin, J. A. Bagnell, and S. Srinivasa, "CHOMP: Covariant hamiltonian optimization for motion planning", The Int. J. of Robotics Research, vol.32, no. 9-10, pp. 1164-1193, August/September 2013.

[19] S. Quinlan, "Real-time Modification of collision-free paths", $\mathrm{PhD}$ thesis, Stanford University, 1994.

[20] I. G. Ramirez-Alpizar, M. Higashimori, M. Kaneko, C.-H. D. Tsai, and I. Kao, "Dynamic Nonprehensile Manipulation for Rotating a Thin Deformable Object: An Analogy to Bipedal Gaits", IEEE Trans. on Robotics, vol. 28, no. 3, pp. 607-618, June 2012. 